\title{
A Neuronal Sec1 Homolog Regulates Neurotransmitter Release at the Squid Giant Synapse
}

\author{
T. Dresbach, ${ }^{1,3}$ M. E. Burns,, ${ }^{2,3}$ V. O'Connor, ${ }^{1}$ W. M. DeBello, ${ }^{2,3}$ H. Betz, ${ }^{1}$ and G. J. Augustine ${ }^{2,3}$ \\ ${ }^{1}$ Department of Neurochemistry, Max-Planck-Institute for Brain Research, 60528 Frankfurt, Germany, ${ }^{2}$ Department of \\ Neurobiology, Duke University Medical Center, Durham, North Carolina 27710, and ${ }^{3}$ Marine Biological Laboratory, Woods \\ Hole, Massachusetts 02543
}

\begin{abstract}
Sec1-related proteins are essential for membrane fusion at distinct stages of the constitutive and regulated secretory pathways in eukaryotic cells. Studies of neuronal isoforms of the Sec1 protein family have yielded evidence for both positive and negative regulatory functions of these proteins in neurotransmitter release. Here, we have identified a squid neuronal homolog (s-Sec1) of Sec1 proteins and examined its function in neurotransmitter release at the squid giant synapse. Microinjection of $\mathrm{s}-\mathrm{Sec} 1$ into the presynaptic terminal of the giant synapse inhibited evoked neurotransmitter release, but this effect was prevented by coinjecting the cytoplasmic domain of squid syntaxin (s-syntaxin), one of the binding partners of s-Sec1. A 24 amino acid peptide fragment of s-Sec1, which
\end{abstract}

Neurotransmission at chemical synapses relies on a cycle of membrane trafficking events that is organized around clusters of synaptic vesicles accumulated at specialized sites of the presynaptic plasma membrane, the active zones. Within these clusters, a subset of vesicles is docked at the active zone membrane and thought to undergo a multistep maturation process during which vesicles acquire competence for $\mathrm{Ca}^{2+}$-triggered exocytosis (Martin, 1997). After exocytotic fusion, the vesicle membrane is retrieved from the plasma membrane by clathrin-mediated endocytosis (Heuser and Reese, 1973; Cremona and De Camilli, 1997). A complex array of protein interactions is proposed to ensure the fidelity of this synaptic vesicle life cycle (Südhof, 1995).

The plasma membrane protein syntaxin 1A plays a central role in neurotransmitter release, and its function appears to be mediated by its interactions with proteins that have been implicated in neurotransmission, including voltage-gated $\mathrm{Ca}^{2+}$ channels (Bennett et al., 1992; Yoshida et al., 1992) and the putative calcium sensor protein synaptotagmin (Südhof and Rizo, 1996), as well as evolutionarily conserved cytosolic proteins essential for membrane fusion termed NSF ( $N$-ethylmaleimide-sensitive fu-

Received Nov. 19, 1997; revised Jan. 29, 1998; accepted Feb. 3, 1998.

This work was supported by Deutsche Forschungsgemeinschaft, Fonds der Chemischen Industrie, National Institutes of Health Grant NS-21624, and a Grass Fellowship to W. DeBello. We are grateful to A. Püschel for providing the mouse Munc18- $a$ cDNA, to A. Niehuis for expert technical assistance, to M. Baier and H. Reitz for secretarial assistance, to L. Bonewald and A. Makusky (University of San Antonio) for peptide synthesis, and to L. Hawkey for electron microscopy.

GenBank accession number is Y12732.

Correspondence should be addressed to Heinrich Betz, Max-Planck-Institute for Brain Research, Neurochemistry Department, Deutschordenstrasse 46, D-60528 Frankfurt/Main, Germany.

Dr. Dresbach's present address: Leibniz Institute for Neurobiology, Department of Neurochemistry and Molecular Biology, Brenneckestrasse 6, D-39118 Magdeburg, Germany.

Copyright (C) 1998 Society for Neuroscience $\quad 0270-6474 / 98 / 182923-10 \$ 05.00 / 0$ inhibited the binding of s-Sec1 to s-syntaxin in vitro, completely blocked release, suggesting an essential function of the $\mathrm{s}-\mathrm{Sec} 1 / \mathrm{s}-\mathrm{syntaxin}$ interaction in transmitter release. Electron microscopy showed that injection of s-Sec1 did not change the spatial distribution of synaptic vesicles at presynaptic release sites ("active zones"), whereas the inhibitory peptide increased the number of docked vesicles. These distinct morphological effects lead us to conclude that Sec1 proteins function at different stages of synaptic vesicle exocytosis, and that an interaction of s-Sec1 with syntaxin-at a stage blocked by the peptide-is necessary for docked vesicles to fuse.

Key words: active zone; microinjection; neurotransmitter release; Sec1 proteins; squid giant synapse; syntaxin

sion protein) and SNAPs (soluble NSF-attachment proteins) (Rothman and Wieland, 1996). The binding of syntaxin 1A to the synaptic vesicle protein synaptobrevin and the plasma membrane protein SNAP-25 (synaptosomal-associated protein of $25 \mathrm{kDa}$ ) results in the formation of a $7 \mathrm{~S}$ complex, termed SNAP-receptor complex (SNARE-complex) because of its ability to recruit SNAPs and NSF. The formation and NSF-catalyzed disassembly of the SNARE-complex are thought to represent functionally important states of the synaptic vesicle docking and fusion reaction (Söllner et al., 1993a,b; Pellegrini et al., 1995; Banerjee et al., 1996; Bock and Scheller, 1997).

Recently, four independent studies have shown that syntaxin also binds to a neuronal protein related to yeast Sec1p, the first protein known to be essential for exocytosis in yeast (Novick and Schekman, 1979; Aalto et al., 1991), and Caenorhabiditis elegans UNC-18 (Gengyo-Ando et al., 1993). This protein has been named Munc18 (Hata et al., 1993), n-Sec1 (Pevsner et al., 1994a), rb-Sec1 (Garcia et al., 1994), and m-Sec1 (Hodel et al., 1994). Detailed in vitro studies using recombinant proteins showed that n-sec1 binds syntaxin with high affinity in a way that prevents binding of SNAP-25 or synaptobrevin and thereby precludes the formation of SNARE-complexes (Pevsner et al., 1994b). This neuronal Sec1 homolog therefore is thought to constitute an important regulator of syntaxin- and SNARE-complex function in vivo.

Although the Sec1 proteins appear to regulate in vitro SNARE complex interactions, their precise in vivo role remains unclear. Overexpression of the Drosophila Sec1 homolog Rop inhibits synaptic transmission, suggesting a negative role of the Rop protein in vivo (Schulze et al., 1994). However, null mutations of Rop or yeast Sec1p also reduce exocytotic activities in the affected cells (Novick and Schekman, 1979; Harrison et al., 1994). 
Furthermore, a mutation in UNC-18 impairs neurotransmission at $C$. elegans neuromuscular junctions (Gengyo-Ando et al., 1993). These findings are inconsistent with a simple inhibitory function of Sec1 proteins. To further elucidate the function and sites of action of Sec1 proteins in neurotransmitter release, we microinjected the squid neuronal Sec1 (s-Sec1) protein and s-Sec1-peptides into the squid giant synapse and analyzed the effects of these agents on synaptic function and ultrastructure. Our results suggest multiple roles of Sec1 proteins in synaptic vesicle exocytosis.

\section{MATERIALS AND METHODS}

Isolation of a squid Sec1 cDNA. A $\lambda$ gt10 squid stellate ganglion cDNA library $\left(10^{6}\right.$ plaque-forming units), kindly provided by J. Battey (National Institutes of Health, Bethesda, MD), was screened with a ${ }^{32} \mathrm{P}-\alpha$-dCTPlabeled $1.6 \mathrm{~kb}$ cDNA probe from the coding region of mouse Munc18-a, kindly provided by A. Püschel (Max-Planck-Institute for Brain Research, Frankfurt). Filter hybridization was performed under low-stringency conditions $\left(56^{\circ} \mathrm{C}\right.$ without formamide), as described previously (Hunt et al., 1994). cDNAs were excised from hybridization-positive clones with EcoRI and subcloned into pBluescript. Sequencing of the largest $(3.2 \mathrm{~kb})$ cDNA isolated revealed a $1773 \mathrm{bp}$ open reading frame preceded by 142 nucleotides and followed by some 1200 nucleotides downstream of the stop codon as mapped by restriction digests.

Generation and purification of fusion proteins. To generate s-Sec1 expression constructs, nucleotides $1-372$ of the open reading frame of the $s$-Sec1 cDNA were amplified by PCR using a sense primer with an artificial BamHI site. After digestion of the PCR product using the Bam HI site and an endogenous HindIII site, the resulting 316 bp DNA was subcloned into the expression vector pQE30 and verified by sequencing. The $3^{\prime}$ coding region of the $s$-Sec 1 cDNA was excised using HindIII and ligated to the $\mathrm{N}$-terminal region in $\mathrm{pQE} 30$, resulting in a construct comprising a His- 6 tag, the entire open reading frame, and the portion of the cDNA downstream of the stop codon. Fusion proteins containing the cytosolic portion of squid syntaxin1 (s-syntaxin) (O'Connor et al., 1997) were generated by subcloning the nucleotides encoding amino acids 2-268 either into pQE30 for N-terminal His-6-tagging or into pGEX$4 \mathrm{~T}-1$ for generation of a glutathione- $S$-transferase (GST) fusion protein. All constructs were expressed in Escherichia coli Xl-I-blue cells, and the resulting fusion proteins were purified under nondenaturing conditions as described previously (Pellegrini et al., 1994).

Affinity purification and in vitro binding assays. For affinity purification of endogenous s-Sec1, frozen optic lobe tissue was homogenized in 25 $\mathrm{mm}$ Tris/ $\mathrm{HCl}, \mathrm{pH} 7.4,0.5 \mathrm{M} \mathrm{KCl}, 0.25 \mathrm{~m}$ sucrose. After centrifugation at $7500 \times g$ for $1 \mathrm{hr}$, the supernatant was cleared for $2 \mathrm{hr}$ at $120,000 \times g$ and subsequently dialyzed against binding buffer $(50 \mathrm{~mm} \mathrm{HEPES} / \mathrm{KOH}, \mathrm{pH}$ $7.4,150 \mathrm{~mm} \mathrm{KCl}, 2 \mathrm{~mm} \beta$-mercaptoethanol). Protease inhibitors from the Complete-cocktail (Boehringer, Mannheim, Germany) were included during these steps. Two milliliters $(10 \mathrm{mg}$ protein) of the dialysate were incubated with $1 \mathrm{nmol}$ GST or GST-s-syntaxin coupled to $50 \mu \mathrm{l}$ glutathione-agarose beads for $4 \mathrm{hr}$. Beads were washed six times with 2 $\mathrm{ml}$ of washing buffer [binding buffer including $0.05 \%(\mathrm{w} / \mathrm{v})$ Tween-20] and eluted with $100 \mu \mathrm{l}$ SDS-PAGE sample buffer. To assay for the binding of recombinant proteins, GST or GST-squid-syntaxin, coupled to $10 \mu \mathrm{l}$ glutathione-agarose beads, was incubated for $2 \mathrm{hr}$ with His-tagged s-Sec1 in a volume of $200 \mu$ l. Beads were washed six times with $500 \mu \mathrm{l}$ washing buffer, eluted with $20 \mu \mathrm{l}$ SDS-PAGE sample buffer, and $10 \mu \mathrm{l}$ aliquots were subjected to SDS-PAGE (Laemmli, 1970).

Microinjection and electrophysiology. Microinjection and electrophysiological recording were performed as described previously (Adler et al., 1991; Hunt et al., 1994). Briefly, three microelectrodes were introduced into stellate ganglia from the squid Loligo pealei. One microelectrode was placed into the presynaptic axon to elicit an action potential once every $30 \mathrm{sec}$. A second microelectrode, introduced into the postsynaptic axon, recorded postsynaptic potentials generated during evoked synaptic transmission. The third electrode, used for both microinjection and recording of presynaptic action potentials, was introduced directly into the presynaptic terminal. Proteins and peptides to be microinjected were dissolved in injection solution $(250 \mathrm{~mm}$ potassium isothionate, $100 \mathrm{~mm}$ taurine, 50 mM HEPES, pH 7.2, $100 \mathrm{~mm} \mathrm{KCl}$ ) containing fluorescein isothiocyanate (FITC)-dextran (100 $\mu \mathrm{M}, 10 \mathrm{kDa})$ (Molecular Probes, Eugene, OR). Reagents were loaded into injection electrodes at the following concentrations: s-Sec1, $7.5 \mu \mathrm{M}$; s-syntaxin, $75 \mu \mathrm{M}$ (for control injections) or 30 $\mu \mathrm{M}$ (for co-injection experiments); peptides, $5 \mathrm{mM}$. To estimate the volume and concentration of the solution injected, FITC fluorescence was measured at regular intervals throughout the experiment and compared with a standard calibration curve obtained from known concentrations of FITC-dextran in microcuvettes in which the path length approximated that of the terminal. To measure presynaptic $\mathrm{Ca}^{2+}$ levels, the fluorescent dye calcium orange (Molecular Probes) was microinjected into the presynaptic terminal using the procedure described by Adler et al. (1991). Dye fluorescence was examined by using a filter set that did not excite FITC-dextran (excitation filter, $546 \mathrm{~nm}$; dichroic mirror, 580 $\mathrm{nm}$; emission filter, $610 \mathrm{~nm}$ ). Video images collected by a sit-camera were stored on an analog optical disk recorder (Panasonic TQ-2026) and analyzed with Image-1 software (Universal Imaging Corporation, West Chester, PA).

Electron microscopy. To assess the morphological effects of microinjected reagent, nerve terminals were fixed and processed for electron microscopy as detailed previously (Bommert et al., 1993; Hunt et al., 1994). Terminals injected with sufficient s-Sec1 (two terminals) or secpep3 (two terminals) to inhibit transmission by $>90 \%$ were compared with 10 control terminals injected with different inactive substances (scrambled secpep3, inert recombinant proteins, or injection buffer alone). Sections were prepared from each terminal at $50 \mu \mathrm{m}$ intervals throughout the active zone region. Each $80 \mathrm{~nm}$ section was magnified 12,000 times, and every active zone was photographed and analyzed, resulting in cumulative data of $\sim 200$ active zones per terminal. Morphometric analysis was performed on the digitized images using Image-1 software.

Miscellaneous methods. SDS-PAGE was performed according to Laemmli (1970). Western blotting and immunodetection using the enhanced chemiluminescence system were performed as detailed previously (Pellegrini et al., 1994). For immunodetection of s-Sec1, a polyclonal antiserum raised against Munc18-1 (Dianova, Hamburg, Germany) was used. Peptides were synthesized by Biosynthesis Inc. (Lewisville, TX) or in the peptide synthesis facility of the University of Texas (San Antonio, TX), and contained alkylated N termini and amidated $\mathrm{C}$ termini.

\section{RESULTS}

\section{Cloning of a squid Sec1 homolog}

To isolate Sec1 cDNAs from squid, we screened a squid stellate ganglion cDNA library under low-stringency conditions using a $1.6 \mathrm{~kb}$ cDNA probe from the coding region of murine Munc18a (A. Püschel, unpublished observations). This procedure led to the isolation of a cDNA clone M21, including an open reading frame of $1773 \mathrm{bp}$ predicted to encode a $67.1 \mathrm{kDa}$ protein with an isoelectric point of 6.76. The deduced amino acid sequence includes consensus sites for phosphorylation by protein kinase C, casein kinase II, calcium/calmodulin kinase, and protein tyrosine kinase (Fig. 1). A homology search using the EMBL/GenBank showed that the protein was closely related to the Sec1/UNC-18 protein family. The squid sequence shared the highest identity with members of the protein family that have been implicated in neurotransmission, i.e., mammalian n-Sec1 (66\%), Drosophila Rop (66\%), and C. elegans UNC-18 (58\%), and was less closely related to the ubiquitously expressed mammalian isoforms Munc18-2/Munc18b (56\%) and Munc18c (46\%). Moreover, the squid protein, referred to as s-Sec1, displayed a higher homology to Sec1p (28\%), the yeast isoform required for exocytosis, than to isoforms that function at other steps in the yeast secretory pathway: Sly1p (23\%), Vps45 (22\%), and Vps33 (21\%). This hierarchy of similarities suggests that s-Sec1 is one of the Sec1/UNC-18 proteins that function in regulated exocytosis. Comparison of s-Sec1 with the sequences of n-Sec1, Rop, and UNC-18 showed that homologies extend over the entire lengths of these proteins (Fig. 1). A centrally located $\beta$-COP-motif, which is conserved among all members of the Sec1/UNC-18 protein family (Halachmi and Lev, 1996), is also found in s-Sec1 (amino acids 226-253). Similarly, the only consensus site for tyrosine phos- 


\begin{tabular}{|c|c|c|}
\hline$s-\sec 1$ & ---MALKTAVHEKIMNDVVLA----VKKN---------AEWKVLIVDQLSMRMVSACCK & 43 \\
\hline$n-\operatorname{Sec} 1$ & 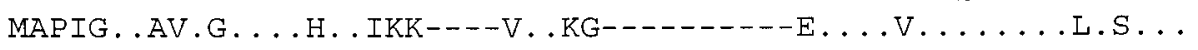 & 46 \\
\hline Rop & $\ldots \ldots$. . VL.GQ.L. . E. KYKPPPP . QGVTSAAGAGGM. .R. .V. . K.G. . . . .T. & 57 \\
\hline UNC-18 & --- .S. .QI.GH.LL . . IRPLK---.GDGR------SA.N. . . . . A . . L.S . . & 46 \\
\hline$s-\operatorname{Sec} 1$ & MHEIMSEGITLVEDINRRREPLPLLEAVYLITPTEESVKCLMADFQNPDNPQYRGAHIFF & 103 \\
\hline$n-\operatorname{Sec} 1$ & 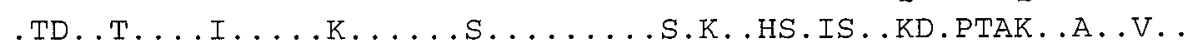 & 106 \\
\hline Rop & 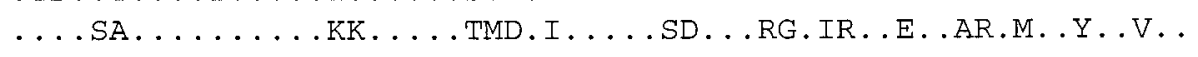 & 117 \\
\hline UNC-18 & 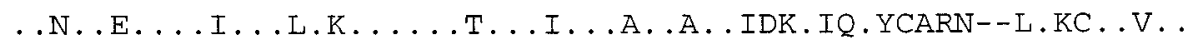 & 104 \\
\hline$s-\operatorname{Sec} 1$ & TEACPEELFKELCKSTTARFIKTLKEINIAFLPYESQIFSLDSPDTFQVYYNPSRAQGGI & 63 \\
\hline $\mathrm{n}-\operatorname{Sec} 1$ & 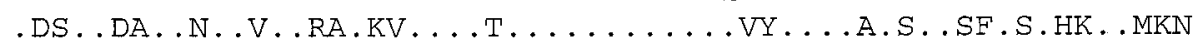 & 66 \\
\hline Rop & $\ldots V \ldots \ldots$ ND . . CA.GK. . . . . . . C.V. . . . CL.S.AF.SIRS & 177 \\
\hline UNC -18 & 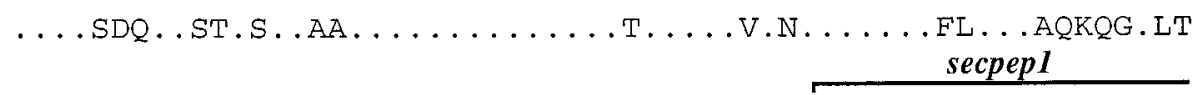 & 164 \\
\hline$s-\operatorname{Sec} 1$ & PNKERCAEQIATLCATLGEYPSVRYRSDFDENASFAQLVQQKLDAYRADDPTMGEGPQKD & 223 \\
\hline$n-\sec 1$ & 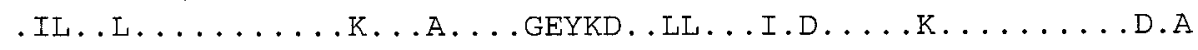 & 226 \\
\hline Rop & 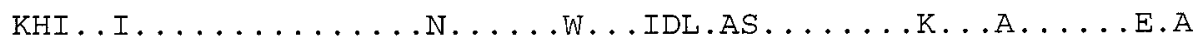 & 237 \\
\hline UNC-18 & 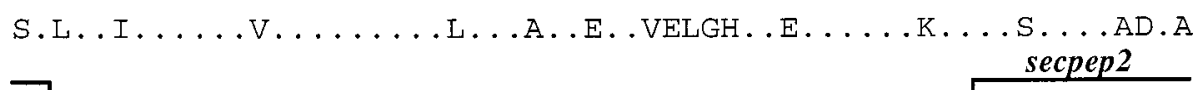 & 224 \\
\hline s-Sec1 & $\overrightarrow{R S Q L L I L D R G F D P I S P L L H E L T F Q A M A Y D L L P I E N D V Y K Y V N T G G N E V P E K E V L V D E K D D ~}$ & 283 \\
\hline $\mathrm{n}-\operatorname{Sec} 1$ & 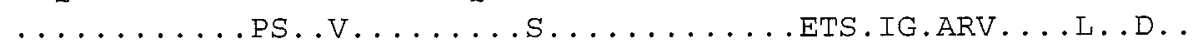 & 286 \\
\hline Rop & 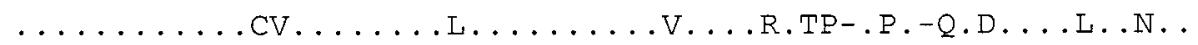 & 295 \\
\hline UNC-18 & 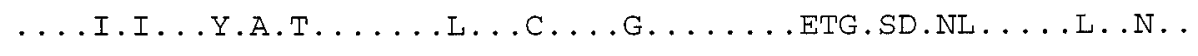 & 284 \\
\hline$s-\sec 1$ & LWVEMRHQHIAVVSQNVTKKLKQFGDEKRMGTAADKAGIKDLSQMLKKMPQYQKELSKYS & 343 \\
\hline$n-\sec 1$ & 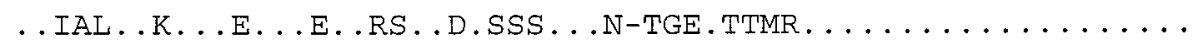 & 345 \\
\hline Rop & 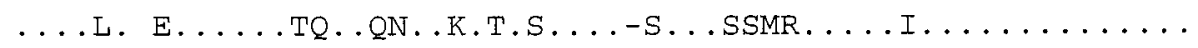 & 354 \\
\hline UNC-18 & $\ldots \ldots \ldots$. . . . E. .N. .K.SES.GNKGTM.SKS . . .MLI.R. . HK . .N.F. & 34 \\
\hline$s-\operatorname{Sec} 1$ & THLHLGEDCMKQYQQHVDKLCKVEQDLAMGTDADGEKIRDHMRNIVPIWLDQKISAYDKI & 40 \\
\hline $\mathrm{n}-\sec 1$ & 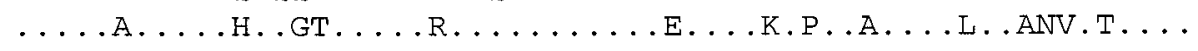 & 405 \\
\hline Rop & 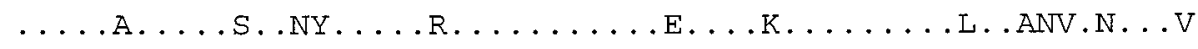 & 414 \\
\hline UNC- 18 & . IS.A.E. . . . . G. . . . . . . ST.I. E. .RV. . A.KLM . LLI. PAVRCE.RL & 404 \\
\hline$s-\sec 1$ & RIILLYI IHKGGISEENLAKLVQHAHIPGEEKWI INDMQNLGVPI IQDGGRRKI PQPYHT & 46 \\
\hline$n-\sec 1$ & 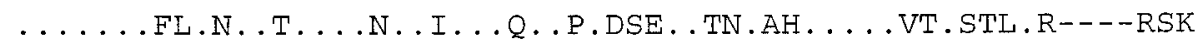 & 461 \\
\hline Rop & . . . . .VMI.N. . . . . . . .FT . QLSPKDQDMVRNLSC . INV.A.S-.-.--KQ.SV & 470 \\
\hline UNC- 18 & 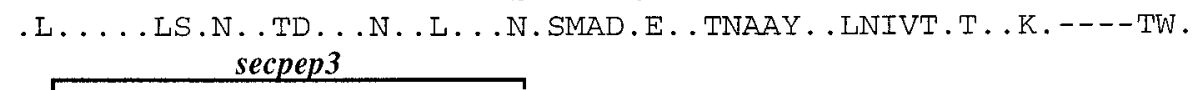 & 460 \\
\hline$s-\operatorname{Sec} 1$ & HNRKERQGDHTYQMSRWTPYMKDIMEAAVEDKLDTRHYPFLNGGGPRPSCQQPVSVRYGH & 523 \\
\hline$n-\operatorname{Sec} 1$ & 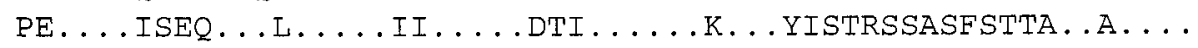 & 521 \\
\hline Rop & 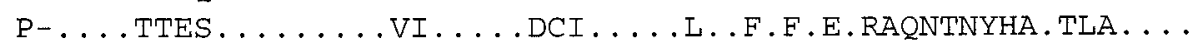 & 529 \\
\hline UNC-18 & PTK...PHEQV. .S...V.VI ...I.D.IDER. . .K.F.F.A.RQVNQGYRA.A.A. . Q & 520 \\
\hline s-Sec1 & WHKDKGQ-ASYKSGPRLIIFVVGGISYSEMRSAYEVTQTAKNNWEVILGSTHILTPEGLL & 582 \\
\hline$n-\sec 1$ & $\ldots$ N.AP-GE.R. . . . . IIL . V.LN . . C . . . . ANGK- . III . . . . . QK . & 579 \\
\hline Rop & 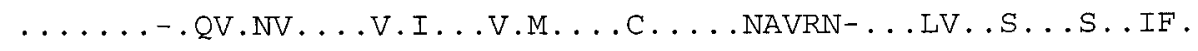 & 587 \\
\hline UNC -18 & $\ldots$ ER . QSN.R. . . . . YII . VTF . . . AC . . . AAR . P- . .VI . DR. I . DKF . & 579 \\
\hline$s-\operatorname{Sec} 1$ & RDLRKISNP------------- & 591 \\
\hline$n-\sec 1$ & DT.K. LNKTDEEISS-NQLPSKTLA & 593 \\
\hline ROP & S. . GSL . KED $-------\cdots \cdots$ & 597 \\
\hline UNC-18 & TN. . DLNK. RDI $\cdots \cdots \cdots$ & 591 \\
\hline
\end{tabular}

Figure 1. Alignment of the deduced amino acid sequences of s-Sec1, n-Sec1, Rop, and UNC-18. Residues identical to the corresponding positions of the squid sequence are indicated by dots. Gaps introduced to optimize the sequence alignment are marked by dashes. Brackets indicate the sequences of s-Sec1 peptides (secpep1, secpep2, secpep3) synthesized for microinjection. Phosphorylation consensus sites in s-Sec1 exist at positions 34, 80, 120, 126, 185, 301, 518, 523 (for protein kinase C), 53, 76, 126, 179, 190, 215, 315, 374, 398, 548 (for casein kinase II), 475 (protein tyrosine kinase), and 589 (for calcium/calmodulin kinase). 
A

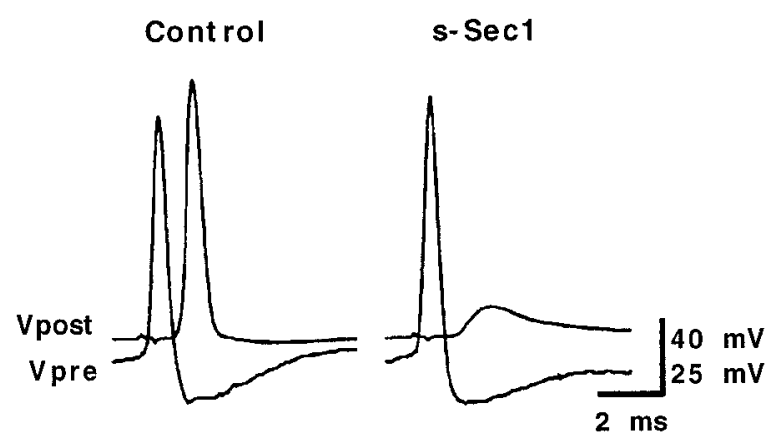

B

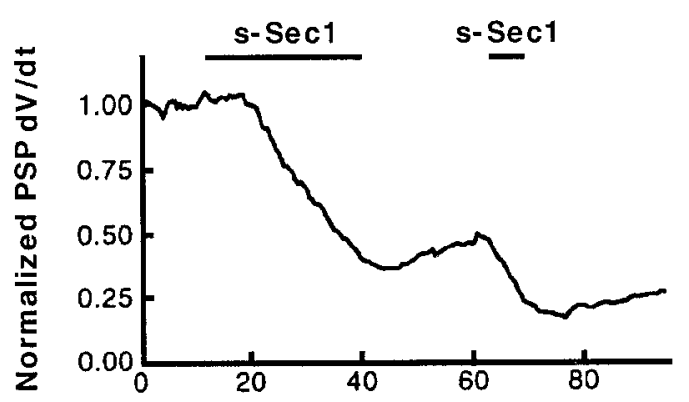

C

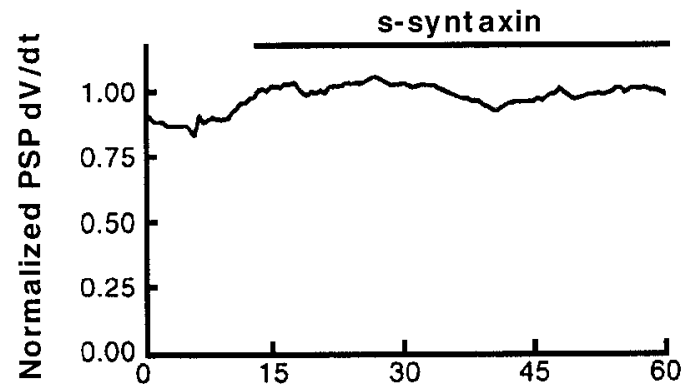

D

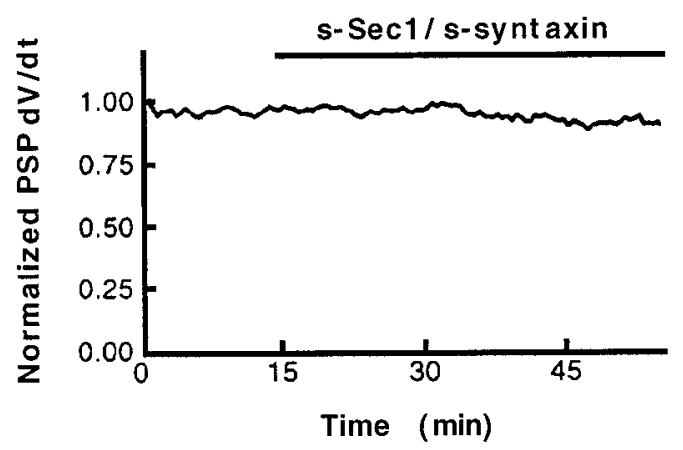

Figure 2. Microinjection of s-Sec1 inhibits neurotransmitter release. $A$, Simultaneous recording of evoked presynaptic action potentials (Vpre) and the resulting postsynaptic responses (Vpost). Microinjection of full length recombinant s-Sec1 inhibited synaptic transmission as was evident from a strong decrease in postsynaptic potential changes (PSPs) without alterations in presynaptic action potentials. $B$, Time course of inhibition of transmitter release by s-Sec1. Microinjection of s-Sec1 during the periods indicated by the bars produced a gradual and partially reversible inhibition of the rate of rise of PSPs. $C$, Microinjection of the cytosolic portion (amino acids 2-268) of squid syntaxin 1 (s-syntaxin) had no effect on transmitter release. $D$, Coinjection of s-syntaxin with s-Sec1 blocked s-Sec1 inhibition of transmitter release. phorylation in s-Sec1, at position 475, corresponds to an evolutionary conserved signature of these proteins.

\section{Effect of recombinant s-Sec1 on neurotransmitter release}

To test whether s-Sec1 is involved in neurotransmitter release, we generated a full length recombinant s-Sec1 construct with an $\mathrm{N}$-terminal histidine tag and purified the bacterial fusion protein under nondenaturing conditions. We then injected the soluble fusion protein into the presynaptic terminal of the squid giant synapse while we electrophysiologically monitored synaptic transmission evoked by single action potentials (Fig. $2 A$ ). s-Sec1 consistently produced an inhibition of neurotransmitter release, as evidenced by the decreasing rate of rise of the evoked postsynaptic potentials (PSPs). There generally was no change in the shape of the presynaptic action potential or the resting potential of the terminal during inhibition of synaptic transmission, indicating that s-Sec1 microinjection affected a step subsequent to arrival of the action potential in the nerve terminal and did not impair the electrical characteristics of the presynaptic terminal. To examine the possibility that s-Sec1 interferes with the rise of calcium levels that trigger release, presynaptic terminals were microinjected with the fluorescent calcium indicator calcium orange (Eberhard and Erne, 1991). Trains of action potentials were then used to elicit calcium signals both before and during inhibition of transmitter release by s-Sec1. Microinjection of s-Sec1 caused no significant change in the magnitude and time course of the $\mathrm{Ca}^{2+}$ fluorescence signal, indicating that s-Sec1 inhibits a step of transmitter release downstream of the calcium signal (Fig. 3).

Inhibition of transmitter release started to reverse within minutes after cessation of s-Sec1 injection and could be reinitiated by resuming injection (Fig. $2 B$ ). This reversibility indicates that the inhibitory effect was not an artifact of microinjection because such artifacts are largely irreversible (Llinas et al., 1991). The comparatively rapid onset of recovery is reminiscent of the reversal of effects of other fusion proteins injected into the giant terminal (Hunt et al., 1994; DeBello et al., 1995) and probably reflects diffusion of the protein out of the terminal and into the giant presynaptic axon (Bommert et al., 1993). With the latter acting as a diffusion sink, even high-affinity protein-protein interactions should undergo disassembly as the concentration of microinjected protein falls below the respective dissociation constant.

To further examine the specificity of s-Sec1 inhibition we performed a series of control injections. Injection of buffer alone had no effect on synaptic transmission (data not shown). Likewise, injection of another His-tagged fusion protein, the cytosolic portion of s-syntaxin (amino acids 2-268) (O'Connor et al., 1997), did not inhibit transmitter release even when present in the injection electrode at a concentration $(75 \mu \mathrm{M})$ an order of magnitude higher than that needed for s-Sec1 to inhibit release (Fig. $2 C$ ). Interestingly, coinjection of s-syntaxin 2-268 together with s-Sec1 at a molar ratio of 4:1 significantly reduced, and in some experiments completely prevented, s-Sec1-inhibition of transmitter release (Fig. 2D, Table 1), suggesting that s-Sec1 was complexed by the syntaxin construct. Together, these experiments corroborate that the inhibitory effect of s-Sec1 injections was not caused by nonspecific effects of the injection procedure or the fusion proteins. Moreover, preventing the inhibitory action of s-Sec1 by s-syntaxin 2-268 is consistent with the idea that s-Sec1 performs its action in the nerve terminal via an interaction with syntaxin. 

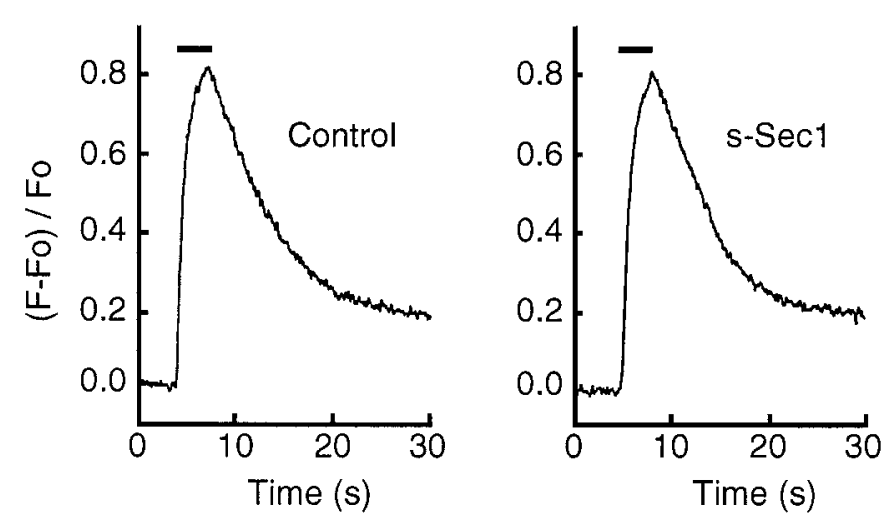

Figure 3. s-Sec1 does not affect presynaptic calcium signaling. Changes in presynaptic calcium concentration produced by trains of action potentials $(50 \mathrm{~Hz}, 3 \mathrm{sec}$ for the time indicated by bars) evoked before (Control) and after $(s-\operatorname{Sec} 1)$ microinjection of s-Sec1 were monitored by measuring changes in fluorescence of the microinjected $\mathrm{Ca}^{2+}$ indicator calcium orange. The plots show the change in calcium orange fluorescence $\left(F-F_{o}\right)$ divided by the prestimulus resting fluorescence $(F o)$.

\section{Identification of active s-Sec1 domains}

Several additional experiments were performed to further determine whether the inhibitory action of s-Sec1 involves an interaction with syntaxin. First, we tested whether endogenous and recombinant s-Sec1 are able to bind syntaxin. We therefore coupled a GST fusion protein that included the cytosolic portion of s-syntaxin or GST alone to glutathione beads to form an affinity matrix. Incubation with the soluble proteins from squid optic lobes lead to purification of a $67 \mathrm{kDa}$ protein that was recognized by an antisera raised against Munc18-1 (Fig. 4A) (and data not shown). Likewise, recombinant s-Sec1 bound to GST-s-syntaxin but not to GST (Fig. 4A). These data show that both endogenous and recombinant s-Sec1 have the ability to interact with s-syntaxin.

We next examined the functional consequences of the interaction of s-Sec1 with syntaxin by using an inhibitory peptide derived from s-Sec1. To this end, we synthesized peptides (secpep1, secpep2, secpep3) from three evolutionarily conserved regions of s-Sec1 that are predicted to be surface-exposed (Fig. 1A). Two of the selected regions have been suggested to be functionally important from studies on Drosophila Rop and yeast Sly1p. The secpep2 peptide covers a domain that contains a conserved histidine residue in positions 290 of s-Sec1 and 302 in Rop. Substitution by tyrosine of this histidine in Rop impairs sensory transduction in Drosophila photoreceptors (Harrison et al., 1994), and introduction of this point mutation into recombinant n-Sec1 inhibits its in vitro binding to syntaxin (Frelin and Pevsner, 1996). Secpep3 aligns with amino acids 526-551 of yeast Sly1p as predicted by the Clustal program (data not shown). This region has been implicated genetically in interactions of Sly1p with GTPbinding protein Ypt1p (Dascher et al., 1991). We then tested whether these peptides affect the in vitro interaction between recombinant s-Sec1 and s-syntaxin. Secpep1 and secpep2 (1 mM) had no effect on the binding of recombinant s-Sec1 to GST-ssyntaxin, whereas secpep3 completely prevented the interaction at the same concentration (Fig. 4B). A dose-response curve revealed half-maximal inhibition at a secpep3 concentration of 75 $\mu \mathrm{M}$ (Fig. 4C).

On the basis of these results we used the s-Sec1 peptides to investigate the possible roles of s-Sec1/s-syntaxin interactions in

\begin{tabular}{lc}
\hline Table 1. Effects of microinjected reagents on neurotransmitter release \\
Reagent & Inhibition $(\%)$ \\
\hline s-Sec1 & $73 \pm 8(13)$ \\
s-Syntaxin & $13 \pm 6(5)$ \\
s-Sec1 + s-syntaxin & $27 \pm 11(6)$ \\
Injection buffer & $5 \pm 1(9)$ \\
Secpep1 & $8 \pm 6(2)$ \\
Secpep2 & $12 \pm 5(8)$ \\
Secpep3 & $57 \pm 11(10)$ \\
scr-Secpep3 & $8 \pm 5(6)$
\end{tabular}

Inhibition of transmitter release was determined after the different agents were microinjected, as detailed in Materials and Methods. The data represent the mean \pm SEM of $n$ (given in parentheses) experiments.

neurotransmitter release. Presynaptic microinjection of either secpep1 or secpep2 had no effect on synaptic transmission; however, the secpep3 peptide produced a rapid and reversible inhibition of transmitter release (Fig. $5 A-C$ ). A high-frequency stimulation paradigm $(3 \mathrm{sec}, 50 \mathrm{~Hz})$ did not change the rate at which release was inhibited during peptide injection, suggesting that replenishment of fused vesicles from the reserve pool of vesicles was not affected (data not shown). To exclude the possibility that the net charge of secpep3 causes the inhibitory effect, we synthesized and injected a scrambled peptide of amino acid composition identical to secpep3 but of randomized sequence (RETKYQMRIGPHYQKMDTMRSWDE). Like secpep1 and secpep2, the scrambled peptide had no effect on transmitter release (Fig. 5D), indicating that the inhibitory effect of secpep3 is sequencespecific. Database searches revealed no significant sequence similarities of secpep3 with proteins other than Sec1 homologs. We therefore conclude that secpep3 inhibits an interaction of s-Sec1 with syntaxin that is essential for neurotransmitter release.

\section{Ultrastructure of synapses inhibited by S-Sec1 or the peptide secpep3}

Neurotransmitter release results from a cycle of membrane trafficking events within presynaptic terminals (Heuser and Reese, 1973; Schweizer et al., 1995). To delineate the trafficking events that are inhibited by s-Sec1 and secpep3, we used quantitative electron microscopy to examine synaptic vesicles in terminals injected with these reagents. Comparison of the morphology of synapses inhibited by s-Sec1 with that of control synapses injected with inert reagents showed that the inhibitory effect of s-Sec1 was not associated with obvious changes in the ultrastructure of the nerve terminal (Fig. 6A). To examine possible effects of s-Sec1 on a quantitative level, we determined the number of synaptic vesicles in concentric shells (bins) of $50 \mathrm{~nm}$ width at increasing distances from the active zone plasma membrane (Hess et al., 1993). The number of vesicles in each of the shells was not changed significantly after synaptic transmission was inhibited by s-Sec1 (Fig. 6B,C). In particular, the number of vesicles in close contact with the plasma membrane, i.e., those within the first 50 $\mathrm{nm}$ bin, was not decreased, indicating that s-Sec1 did not impair docking of synaptic vesicles at the release sites. Furthermore, there was no change in the number of vesicles located more distant $(>50 \mathrm{~nm})$ from the plasma membrane; these vesicles are thought to constitute the reserve pool derived from the endocytotic branch of the vesicle cycle (Pieribone et al., 1995). Thus, s-Sec1 inhibits synaptic transmission by blocking a step of the release process that follows vesicle docking.

We next considered the morphological effects of perturbing 
A
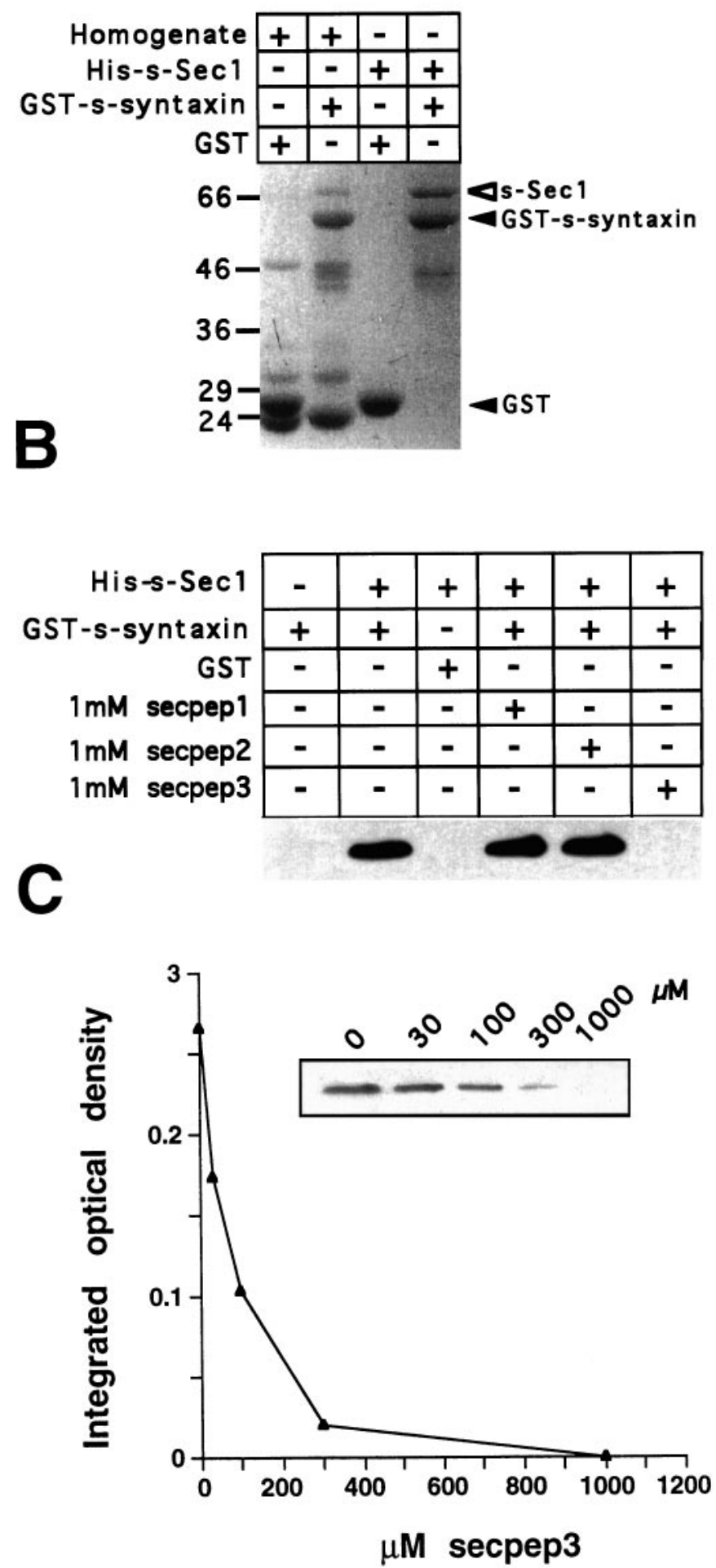

Figure 4. The s-Sec1-peptide secpep3 inhibits binding of s-Sec1 to s-syntaxin. $A$, The soluble fraction of a squid optic lobe homogenate or recombinant s-Sec1 was incubated with GST or GST-s-syntaxin coupled to glutathione-agarose beads. Eluates from the washed beads were analyzed by SDS-PAGE followed by Coomassie blue R250-staining. A 67 $\mathrm{kDa}$ protein from squid homogenate and recombinant s-Sec1 were recovered from GST-s-syntaxin beads but not from GST beads. B, GST or GST-s-syntaxin were coupled to glutathione-agarose beads and incubated with recombinant s-Sec1 in the presence of $1 \mathrm{~mm}$ secpep1, secpep2,
s-Sec1 binding to syntaxins by comparing synapses, in which release was inhibited by $>90 \%$ (as in Materials and Methods) after secpep3 injection, with those injected with the inert scrambled version of secpep3 (scr-secpep3; Fig. 6A). Synapses inhibited by secpep 3 showed no gross change in presynaptic morphology; however, some vesicles were coated with an electron-dense material (Fig. 6A). Although secpep3 did not lead to formation of electron-dense coats when incubated with purified synaptic vesicles in the presence of squid cytosol (V. O'Connor and W. Hofer, unpublished observations), we cannot exclude the possibility that the peptide can attach to vesicles when microinjected into the nerve terminal. Possible attachment sites could be provided by syntaxin; transfection of syntaxin into mammalian cells induces membrane localization of a Sec1 homolog (Riento et al., 1996), and some syntaxin is associated with synaptic vesicles in rat (Walch-Solimena et al., 1995) and squid (V. O'Connor and L. L. Pellegrini, unpublished observations). However, coating per se is unlikely to account for inhibition of transmitter release because inhibition by secpep3 was readily reversible (Fig. 4). This reversibility excludes the possibility that the coat represents precipitated peptide that unspecifically damages the release machinery. Moreover, because secpep3 did not lead to coating of mitochondria and the control peptide did not cause any coat formation, these morphological changes appear to be a specific consequence of secpep3 injection. Finally, quantitative analysis of the spatial distribution of vesicles at active zones revealed that the number of vesicles in almost every bin was similar in both control and inhibited synapses (Fig. 6B,C). Thus, basic processes such as vesicle recycling and maintenance of vesicle clusters at active zones were not affected by peptide injection, indicating that the coating material is not simply preventing synaptic vesicles from interacting with other proteins. However, the number of vesicles within the first $50 \mathrm{~nm}$ compartment, representing the location of docked vesicles, was increased twofold (Fig. 6B,C). Similar effects have been observed after injection of inhibitory peptides derived from other proteins (Bommert et al., 1993; DeBello et al., 1995) and have been interpreted as resulting from the blocking of a reaction that becomes important after vesicle docking, leading to an accumulation of docked vesicles that cannot fuse. Thus, although both s-Sec1 and secpep3 inhibit transmitter release, we observed both qualitative and quantitative differences, which suggests that these reagents disrupt distinct reactions in synapic vesicle traffic.

\section{DISCUSSION}

Genetic and biochemical approaches have implicated members of the Sec1 protein family in secretion in both neuronal and nonneuronal cells. However, the interactions that Sec1 proteins undergo in vivo and the roles these proteins play in exocytosis have remained controversial (Halachmi and Lev, 1996). Particular questions that have remained open include the following. (1) Do neuronal Sec1 proteins play inhibitory, essential, or multiple roles

$\leftarrow$

or secpep3, each. Eluates from washed beads were analyzed by SDSPAGE followed by immunoblotting for s-Sec1. Secpep3, but not secpep1 or secpep2, prevented the binding of s-Sec1 to GST-squid-syntaxin. $C$ GST-s-syntaxin coupled to glutathione-agarose beads was incubated with $\mathrm{s}-\mathrm{Sec} 1$ in the presence of $0,30,100,300$, or $1000 \mu \mathrm{M}$ secpep3. Eluates from the washed beads were analyzed as in $B$, the resulting bands (inset) were quantified densitometrically, and after background correction, integrated optical density peaks were plotted against secpep3 concentrations. The data indicate half-maximal inhibition at $\sim 75 \mu \mathrm{M}$ secpep 3 . 
A

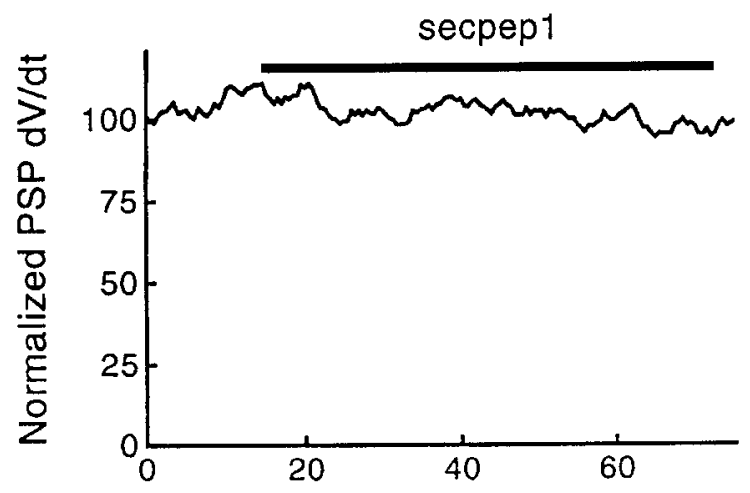

B
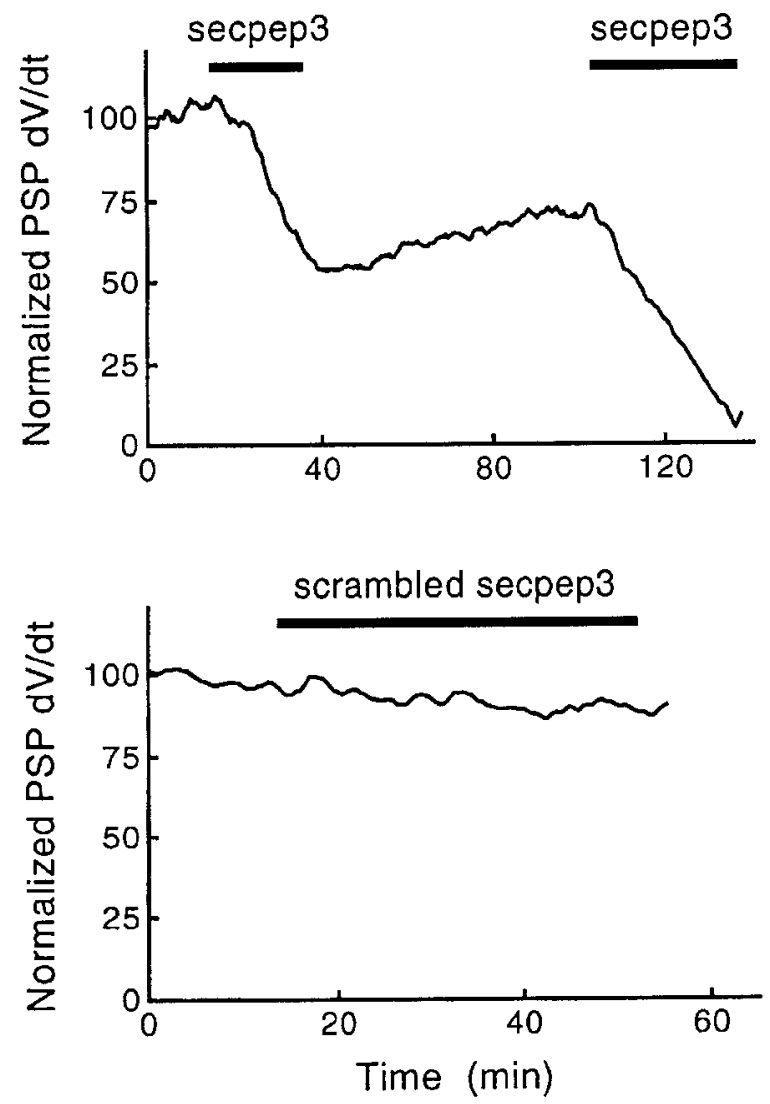

Figure 5. Peptide secpep3 inhibits evoked neurotransmitter release. Secpep3 $(B)$ produced a gradual and reversible inhibition of transmitter release, whereas secpep1 $(A)$ and scrambled secpep3 $(C)$ had no effect. The rates of rise of PSPs, normalized relative to the mean preinjection value, are plotted, with the times of injection indicated by bars.

in synaptic transmission? (2) Is an interaction with syntaxin important in any such function in an intact synapse? (3) To which steps in the synaptic vesicle life cycle can Sec1 protein functions be assigned? To address these questions we have used the squid giant synapse, which allows the combined use of electrophysiological recording of synaptic transmission and ultrastructural analysis of a large number of synaptic contacts with microinjection of probes directly into the giant presynaptic terminal. The results presented here suggest that $\mathrm{s}-\mathrm{Sec} 1$ functions at different stages after synaptic vesicles dock at the plasma membrane, and that at least one of these functions involves an interaction with syntaxin.

To allow an analysis of Sec1 protein function at the squid giant synapse, we first cloned the squid homolog of Sec1p. s-Sec1 is most closely related to rat n-Sec1, Drosophila Rop, and C. elegans UNC-18, Sec1 proteins that have been implicated in neurotransmission. s-Sec1, n-Sec1, and Rop are equally related to one another, with amino acid identities of $66 \%$. This similarity is the same as that between squid SNAP and mammalian $\alpha$-SNAP, proteins for which functional conservation has been demonstrated (DeBello et al., 1995). Therefore, it seems likely that s-Sec1 represents a functional homolog of n-Sec1/Rop type Sec1 proteins. We have chosen the name s-Sec1 to account for the fact that yeast Sec1 was the first member of this growing protein family to be cloned and sequenced (Aalto et al., 1991).

\section{Multiple roles of s-Sec1 in neurotransmission?}

Microinjection of recombinant s-Sec1 into the presynaptic terminal of the squid giant synapse produced a profound inhibition of neurotransmitter release evoked by action potentials. This result is in accord with inhibition of synaptic transmission at Drosophila neuromuscular junctions after chronic overexpression of Rop (Schulze et al., 1994). The fact that we observed similar inhibitory effects after acute injection of s-Sec1 argues that both manipulations work via direct, Sec1-related effects on synaptic transmission. Moreover, s-Sec1 did not affect the amplitude or time course of presynaptic action potentials and calcium signals. Hence, our data extend earlier work by showing that s-Sec1 inhibits a step of transmitter release that lies downstream of action potential propagation and calcium influx.

To reconcile the opposing effects of overexpression and loss of function of Rop and other Sec1 proteins on secretion, it has been suggested that Sec1 proteins exert multiple roles, including inhibitory and stimulatory functions (Pevsner, 1996). Assuming that inhibition by Sec1 proteins precedes their stimulatory role, increased levels of Sec1 might only produce inhibition, as observed on s-Sec1 injection (this study) and overexpression of Rop (Schulze et al., 1994). Conversely, disruption of sec-1 function might unravel subsequent effects that promote exocytosis. Assuming that syntaxin participates in Sec1 protein-mediated effects, we first verified that the syntaxin-Sec1 protein interaction is conserved in squid nervous tissue extracts. Our observation that peptide secpep3 disrupted not only this interaction in vitro but effectively blocked synaptic transmission in vivo, whereas other Sec1 peptides were ineffective in both cases, strongly supports an essential role of s-syntaxin/s-Sec1-interactions in the transmitter release process. This is consistent with a preliminary report showing that point mutations of $\mathrm{n}-\mathrm{Sec} 1$ that mimic amino acid exchanges in loss of function mutations of Drosophila Rop interfere with syntaxin binding in vitro (Frelin and Pevsner, 1996).

It has been proposed that in yeast endoplasmic reticulum-toGolgi transport, the Sec1p homolog Sly1p acts on the syntaxin homolog Sed5p to both activate it for and prevent it from forming SNARE-complexes (Lupashin and Waters, 1997). On the basis of this idea, both negative and permissive Sec1 protein functions would involve syntaxin. Indeed, coinjection of the cytosolic portion of squid syntaxin prevented s-Sec1 inhibition of transmitter release, whereas the syntaxin fragment alone had no effect. Thus, block of inhibition did not result from an independent stimulatory effect of squid syntaxin, but instead from s-syntaxin binding to s-Sec1, consistent with s-Sec1 inhibition resulting from titration of cellular syntaxin. However, it is also possible that the two recom- 

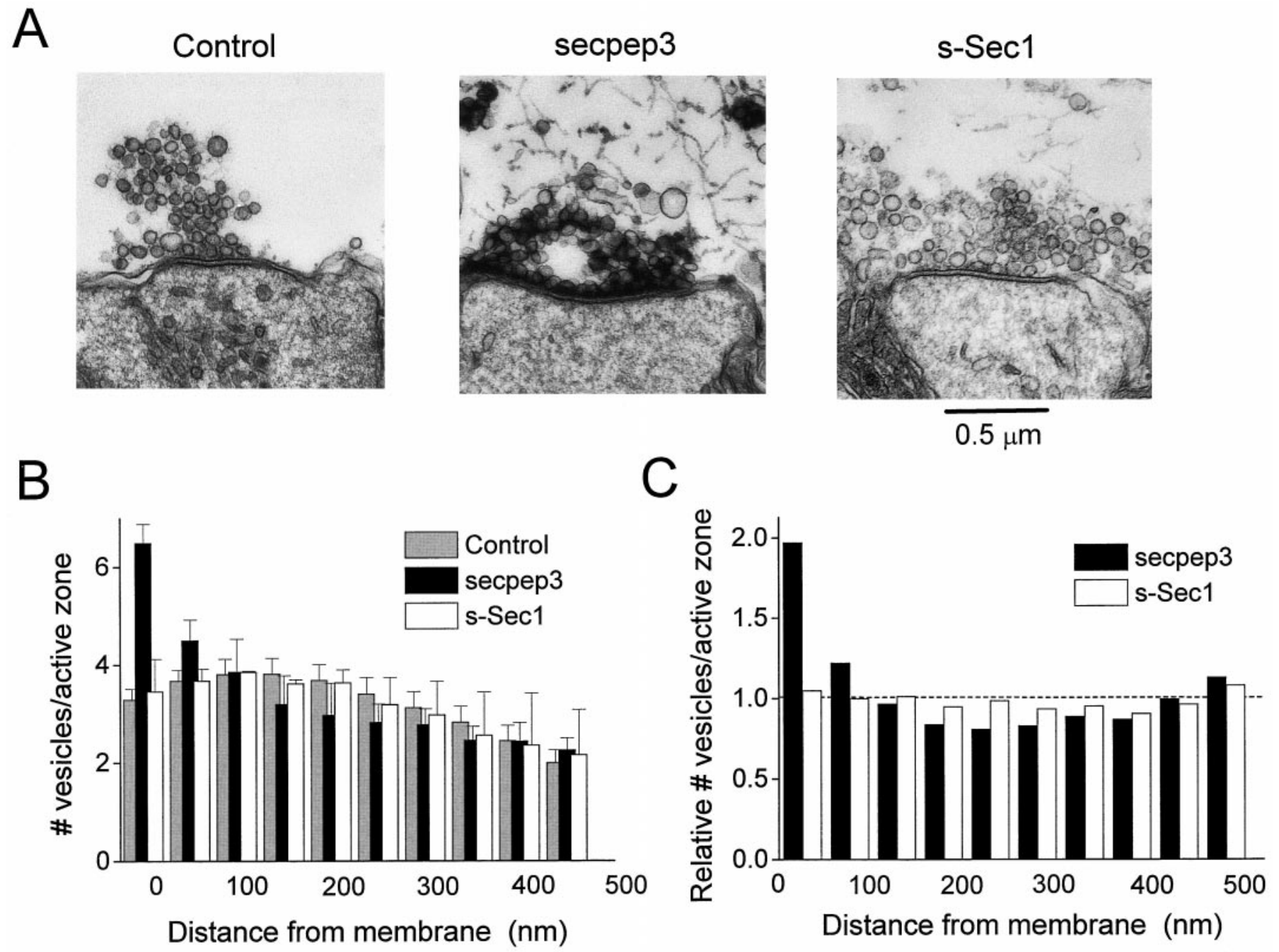

Figure 6. Distinct effects of s-Sec1 and secpep3 on synaptic ultrastructure and the spatial distribution of synaptic vesicles. $A$, Electron micrographs of representative active zones from a control nerve terminal (left) and from terminals fixed during inhibition through secpep3 (middle) or s-Sec1 (right). Secpep3 inhibition was accompanied by coating of synaptic vesicles with electron-dense material (middle), whereas s-Sec1 inhibition did not cause any observable changes in terminal ultrastructure. $B$, Mean number of synaptic vesicles located at various distances from active zone membranes in nerve terminals inhibited by control (solid bars), secpep3 (gray bars), and s-Sec1 (open bars). Inhibition caused by secpep3 was characterized by an increased number of docked vesicles. Terminals inhibited by s-Sec1 showed no change in the distribution of synaptic vesicles as compared with control terminals. $C$, Comparison of the spatial distributions of synaptic vesicles in terminals inhibited by secpep3 ( gray bars) and s-Sec1 (open bars). Inhibition by secpep3 was characterized by an almost twofold increase in the number of docked synaptic vesicles and a decrease in the number of vesicles located a distance of between 100 and $400 \mathrm{~nm}$ from active zones as compared with terminals inhibited by s-Sec1. Numbers are derived from the data shown in $B$.

binant proteins form a complex that prevents s-Sec1 from interacting with other proteins. Possible candidates for such interactions include the recently discovered DOC2 proteins, which compete with syntaxin for $\mathrm{n}$-Sec1 binding in vitro (Verhage et al., 1997), as well as other Sec1-binding partners such as cdk5 (Shetty et al., 1995). This latter interpretation, which invokes independent negative and permissive s-Sec1 functions, receives support from our observation that the inhibitory peptide secpep 3 caused an accumulation of docked vesicles at the active zone membrane and full length s-Sec1 did not. Therefore, this domain of s-Sec1 must have arrested transmitter release at a step distinct from that blocked by the full length protein. Although an accumulation of docked vesicles could result from disruption of an s-Sec1/ssyntaxin interaction that inhibits vesicle docking (Pevsner et al., 1994a), the fact that the peptide blocked neurotransmitter release, rather than leaving it unaffected or enhancing it, is inconsistent with this possibility. Moreover, our data show that both
s-Sec1 and the inhibitory peptide secpep3 did not perturb the clustering of synaptic vesicles at active zones, a process that is sensitive to both synapsin antibodies (Pieribone et al., 1995) and a SNAP peptide (DeBello et al., 1995). Also, neither treatment prevented targeting to and anchoring of vesicles at the active zone membrane. These findings suggest strongly that inhibition by both s-Sec1 and secpep3 results from disruption of reactions that follow synaptic vesicle docking but precede exocytosis. This is consistent with studies on the role of syntaxin, in which both toxin cleavage and gene deletion failed to significantly change the number of docked vesicles (Broadie et al., 1995; O'Connor et al., 1997). We therefore propose that s-Sec1 has at least two functions: (1) a positive role in exocytosis that requires binding to s-syntaxin, is prevented by secpep3, and is needed for docked vesicles to fuse, and (2) a negative role that is prevented by providing an excess of s-syntaxin and also occurs after docking but upstream of the action mediated by the secpep3 domain. 
An indication of how s-Sec1 functions might be regulated comes from the fact that the secpep3 sequence aligns with a region in yeast Sly1p that has been implicated in genetic interactions with Ypt1p (Dascher et al., 1991), a monomeric GTPase essential for SNARE complex assembly in yeast (Sögard et al., 1994; Lupashin and Waters, 1997). A point mutation in this region of Sly1p renders the protein capable of restoring SNARE complex assembly and secretion in the absence of Ypt1p (Dascher et al., 1991; Lupashin and Waters, 1997), suggesting that the mutated Sly1p has a conformation required for proper vesicle traffic that the wild-type Sly1p adopts only after induction by Ypt1p (Dascher et al., 1991). Because secpep3 inhibits the s-Sec1/ s-syntaxin interaction, one may infer that this domain of s-Sec1 could be both subject to regulation by GTPases, such as the squid neuronal Rab3A (Chin and Goldman, 1992; Burns et al., 1995), and involved in syntaxin binding, thus providing a site for crosstalk between the three proteins. Further dissection of Sec1 protein functions, in particular identification of the molecular interactions that specify the negative and positive roles of these proteins, will be required to elucidate the precise roles that Sec1 proteins play in both constitutive and regulated membrane fusion.

\section{REFERENCES}

Aalto MK, Ruohonen L, Hosono K, Keranen S (1991) Cloning and sequencing of the yeast Saccharomyces cerevisiae SEC1 gene localized on chromosome IV. Yeast 7:643-650.

Adler EM, Augustine GJ, Duffy SN, Charlton MP (1991) Alien intracellular calcium chelators attenuate neurotransmitter release at the squid giant synapse. J Neurosci 11:1496-1507.

Banerjee A, Barry VA, DasGupta BR, Martin TFJ (1996) $N$-Ethylmaleimide-sensitive factor acts at a prefusion ATP-dependent step in Ca2+-activated exocytosis. J Biol Chem 271:20223-20226.

Bennett MK, Calakos N, Scheller R (1992) Syntaxin: a synaptic protein implicated in docking of synaptic vesicles at presynaptic active zones. Science 257:255-259.

Bock JB, Scheller RH (1997) Protein transport. A fusion of new ideas. Nature 387:133-135.

Bommert K, Charlton MP, DeBello WM, Chin GJ, Betz H, Augustine GJ (1993) Inhibition of neurotransmitter release by C2-domain peptides implicates synaptotagmin in exocytosis. Nature 363:163-165.

Broadie K, Prokop A, Bellen HJ, O'Kane CJ, Schulze KL, Sweeney ST (1995) Syntaxin and synaptobrevin function downstream of vesicle docking in Drosophila. Neuron 15:663-673.

Burns ME, Beushausen SA, Chin GJ, Tang D, DeBello WM, Dresbach T, O'Connor V, Schweizer FE, Wang SS, Whiteheart SW (1995) Proteins involved in synaptic vesicle docking and fusion. Cold Spring Harb Symp Quant Biol 60:337-348.

Chin GJ, Goldman SA (1992) Purification of squid synaptic vesicles and characterization of the vesicle-associated proteins synaptobrevin and Rab3A. Brain Res 571:89-96.

Cremona O, De Camilli P (1997) Synaptic vesicle endocytosis. Curr Opin Neurobiol 7:323-330.

Dascher C, Ossig R, Gallwitz D, Schmitt HD (1991) Identification and structure of four yeast genes (SLY) that are able to suppress the functional loss of YPT1, a member of the RAS superfamily. Mol Cell Biol 11:872-885.

DeBello WM, O'Connor V, Dresbach T, Whiteheart SW, Wang SS, Schweizer FE, Betz H, Rothman JE, Augustine GJ (1995) SNAPmediated protein-protein interactions essential for neurotransmitter release. Nature 373:626-630.

Eberhard M, Erne P (1991) Calcium binding to fluorescent calcium indicators: calcium green, calcium orange and calcium crimson. Biochem Biophys Res Commun 180:209-215.

Frelin L, Pevsner J (1996) Characterization of N-sec1 binding to syntaxin suggests a positive role in secretion. Soc Neurosci Abstr 22:777.

Garcia EP, Gatti E, Butler M, Burton J, De Camilli P (1994) A rat brain Sec1 homologue related to Rop and UNC18 interacts with syntaxin. Proc Natl Acad Sci USA 91:2003-2007.

Gengyo-Ando K, Kamiya Y, Yamakawa A, Kodaira K, Nishiwaki K,
Miwa J, Hori I, Hosono R (1993) The C. elegans unc-18 gene encodes a protein expressed in motor neurons. Neuron 11:703-711.

Halachmi N, Lev Z (1996) The Sec1 family: a novel family of proteins involved in synaptic transmission and general secretion. $\mathbf{J}$ Neurochem 66:889-897.

Harrison SD, Broadie K, van de Goor J, Rubin GM (1994) Mutations in the Drosophila Rop gene suggest a function in general secretion and synaptic transmission. Neuron 13:555-566.

Hata Y, Slaughter CA, Sudhof TC (1993) Synaptic vesicle fusion complex contains unc-18 homologue bound to syntaxin. Nature 366:347-351.

Hess SD, Doroshenko PA, Augustine GJ (1993) A functional role for GTP-binding proteins in synaptic vesicle cycling. Science 259: 1169-1172.

Heuser JE, Reese TS (1973) Evidence for recycling of synaptic vesicle membrane during transmitter release at the frog neuromuscular junction. J Cell Biol 57:315-344.

Hodel A, Schafer T, Gerosa D, Burger MM (1994) In chromaffin cells, the mammalian Sec1p homologue is a syntaxin 1A- binding protein associated with chromaffin granules. J Biol Chem 269:8623-8626.

Hunt JM, Bommert K, Charlton MP, Kistner A, Habermann E, Augustine GJ, Betz H (1994) A post-docking role for synaptobrevin in synaptic vesicle fusion. Neuron 12:1269-1279.

Laemmli UK (1970) Cleavage of structural proteins during the assembly of the head of bacteriophage T4. Nature 227:680-685.

Llinas R, Gruner JA, Sugimori M, McGuinness TL, Greengard P (1991) Regulation by synapsin I and $\mathrm{Ca}(2+)$-calmodulin-dependent protein kinase II of the transmitter release in squid giant synapse. J Physiol (Lond) 436:257-282.

Lupashin VV, Waters MG (1997) t-SNARE activation through transient interaction with a rab-like guanosine triphosphatase. Science 276:1255-1258.

Martin TFJ (1997) Stages of regulated exocytosis. Trends Cell Biol 7:271-276.

Novick P, Schekman R (1979) Secretion and cell-surface growth are blocked in a temperature-sensitive mutant of Saccharomyces cerevisiae. Proc Natl Acad Sci USA 76:1858-1862.

O'Connor V, Heuss C, DeBello WM, Dresbach T, Charlton MP, Hunt JH, Pellegrini LL, Hodel A, Burger MM, Betz H, Augustine GJ, Schaefer T (1997) Disruption of syntaxin-mediated protein interactions blocks neurotransmitter secretion. Proc Natl Acad Sci USA 94:12186-12191.

Pellegrini LL, O’Connor V, Betz H (1994) Fusion complex formation protects synaptobrevin against proteolysis by tetanus toxin light chain. FEBS Lett 353:319-323.

Pellegrini LL, O’Connor V, Lottspeich F, Betz H (1995) Clostridial neurotoxins compromise the stability of a low energy SNARE complex mediating NSF activation of synaptic vesicle fusion. EMBO J 14:4705-4713.

Pevsner J (1996) The role of Sec1p-related proteins in vesicle trafficking in the nerve terminal. J Neurosci Res 45:89-95.

Pevsner J, Hsu SC, Scheller RH (1994a) n-Sec1: a neural-specific syntaxin-binding protein. Proc Natl Acad Sci USA 91:1445-1449.

Pevsner J, Hsu SC, Braun JE, Calakos N, Ting AE, Bennett MK, Scheller RH (1994b) Specificity and regulation of a synaptic vesicle docking complex. Neuron 13:353-361.

Pieribone VA, Shupliakov O, Brodin L, Hilfiker-Rothenfluh S, Czernik AJ, Greengard P (1995) Distinct pools of synaptic vesicles in neurotransmitter release. Nature 375:493-497.

Riento K, Jantti J, Jansson S, Hielm S, Lehtonen E, Ehnholm C, Keranen S, Olkkonen VM (1996) A sec1-related vesicle-transport protein that is expressed predominantly in epithelial cells. Eur J Biochem 239:638-646.

Rothman JE, Wieland FT (1996) Protein sorting by transport vesicles. Science 272:227-234.

Schulze KL, Littleton JT, Salzberg A, Halachmi N, Stern M, Lev Z, Bellen HJ (1994) rop, a Drosophila homolog of yeast Sec1 and vertebrate $\mathrm{n}-\mathrm{Sec} 1 /$ Munc-18 proteins, is a negative regulator of neurotransmitter release in vivo. Neuron 13:1099-1108.

Schweizer FE, Betz H, Augustine GJ (1995) From vesicle docking to endocytosis: intermediate reactions of exocytosis. Neuron 14:689-696.

Shetty KT, Kaech S, Link WT, Jaffe H, Flores CM, Wray S, Pant HC, Beushausen S (1995) Molecular characterization of a neuronalspecific protein that stimulates the activity of Cdk5. J Neurochem 64:1988-1995. 
Sögaard M, Tani K, Ye RR, Geromanos S, Tempst P, Kirchhausen T, Rothman JE, Söllner T (1994) A rab protein is required for the assembly of SNARE complexes in the docking of transport vesicles. Cell 78:937-948.

Söllner T, Whiteheart SW, Brunner M, Erdjument-Bromage $\mathrm{H}$, Geromanos S, Tempst P, Rothman JE (1993a) SNAP receptors implicated in vesicle targeting and fusion. Nature 362:318-324.

Söllner T, Bennett MK, Whiteheart SW, Scheller RH, Rothman JE (1993b) A protein assembly-disassembly pathway in vitro that may correspond to sequential steps of synaptic vesicle docking, activation, and fusion. Cell 75:409-418.

Südhof TC (1995) The synaptic vesicle cycle: a cascade of proteinprotein interactions. Nature 375:645-653.
Südhof TC, Rizo J (1996) Synaptotagmins: C2-domain proteins that regulate membrane traffic. Neuron 17:379-388.

Verhage M, de Vries KJ, Roshol H, Burbach JP, Gispen WH, Sudhof TC (1997) DOC2 proteins in rat brain: complementary distribution and proposed function as vesicular adapter proteins in early stages of secretion. Neuron 18:453-461.

Walch-Solimena C, Blasi J, Edelmann L, Chapman ER, von Mollard GF, Jahn R (1995) The t-SNAREs syntaxin 1 and SNAP-25 are present on organelles that participate in synaptic vesicle recycling. J Cell Biol 128:637-645.

Yoshida A, Oho C, Omori A, Kuwahara R Ito T, Takahashi M (1992) HPC-1 is associated with synaptotagmin and $\omega$-conotoxin receptor. J Biol Chem 267:24925-24928. 